Supplementary Documents

\title{
Use of Modified SIRD Model to analyze COVID-19 Data
}

\author{
Devosmita Sen ${ }^{1}$, Debasis Sen ${ }^{2,3^{*}}$ \\ ${ }^{1}$ Department of Chemical Engineering, Indian Institute of Technology, \\ Bombay, Mumbai- 400 076, India \\ 2 Solid State Physics Division, Bhabha Atomic Research Centre, Mumbai- 400085, India \\ ${ }^{3}$ Homi Bhabha National Institute, Anushaktinagar, Mumbai- 400094, India
}

Devosmita Sen: $4^{\text {th }}$ year B. Tech. student at IIT, Bombay

Email: devosmita.sen@gmail.com, 170020021@,iitb.ac.in, debasis@barc.gov.in
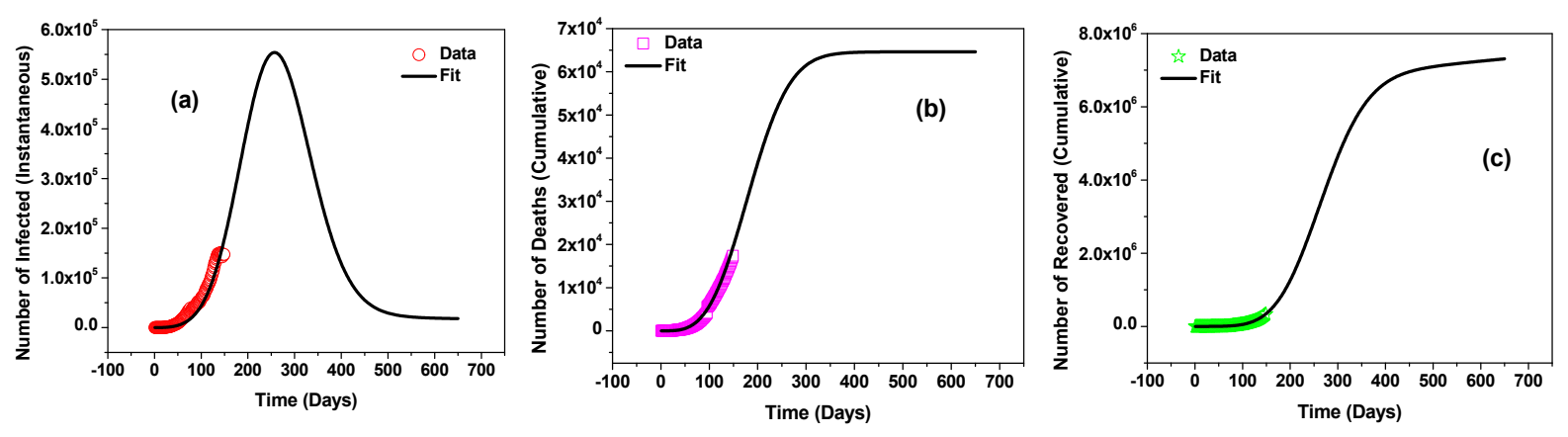

Fig.S1.: (a) Number of active cases of infection in Maharashtra state of India vs. time in days (b) Cumulative number of deaths due to infection vs. time in days (c) Cumulative number of people recovered from infection vs. time in days. Solid lines represent the model (modified SIRD) fit to the data 

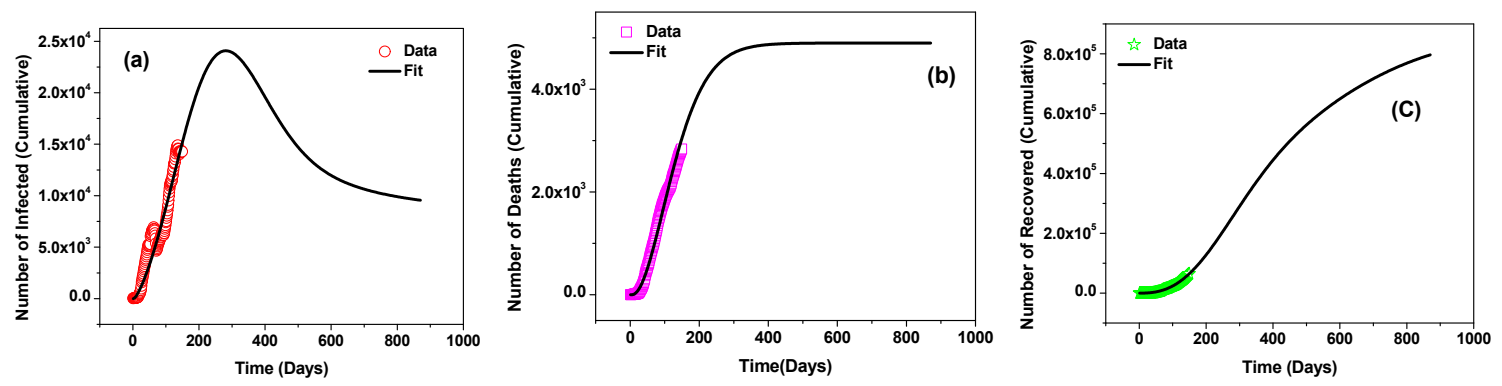

Fig.S2.: (a) Number of active cases of infection in Gujarat state of India vs. time in days (b) Cumulative number of deaths due to infection vs. time in days (c) Cumulative number of people recovered from infection vs. time in days. Solid lines represent the model (modified SIRD) fit to the data
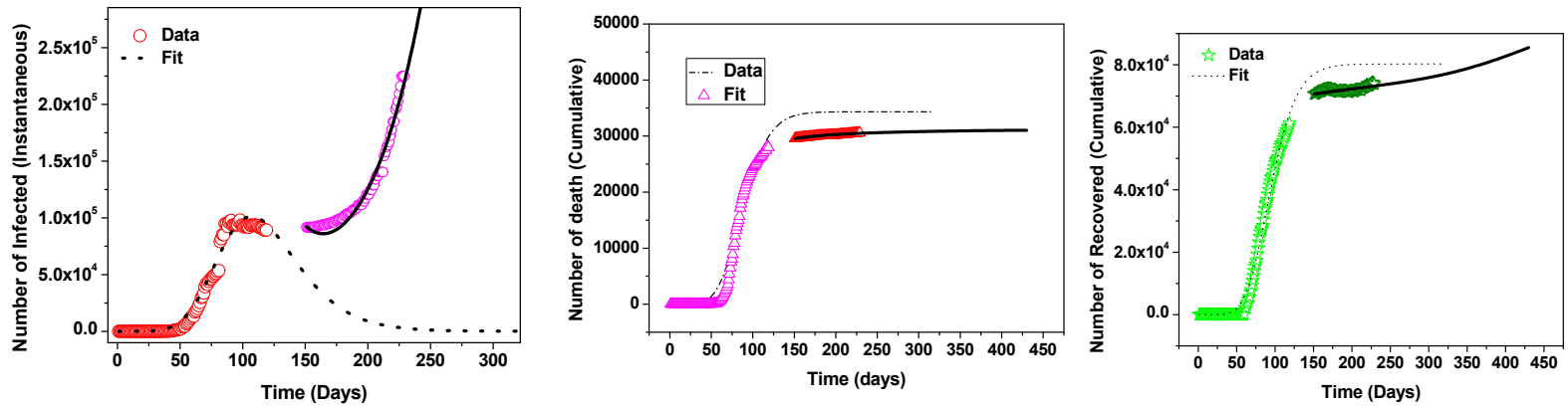

Fig.S3.: (a) Number of active cases of infection in France vs. time in days (b) Cumulative number of deaths due to infection vs. time in days (c) Cumulative number of people recovered from infection vs. time in days. Solid lines represent the model (modified SIRD) fit to the data.
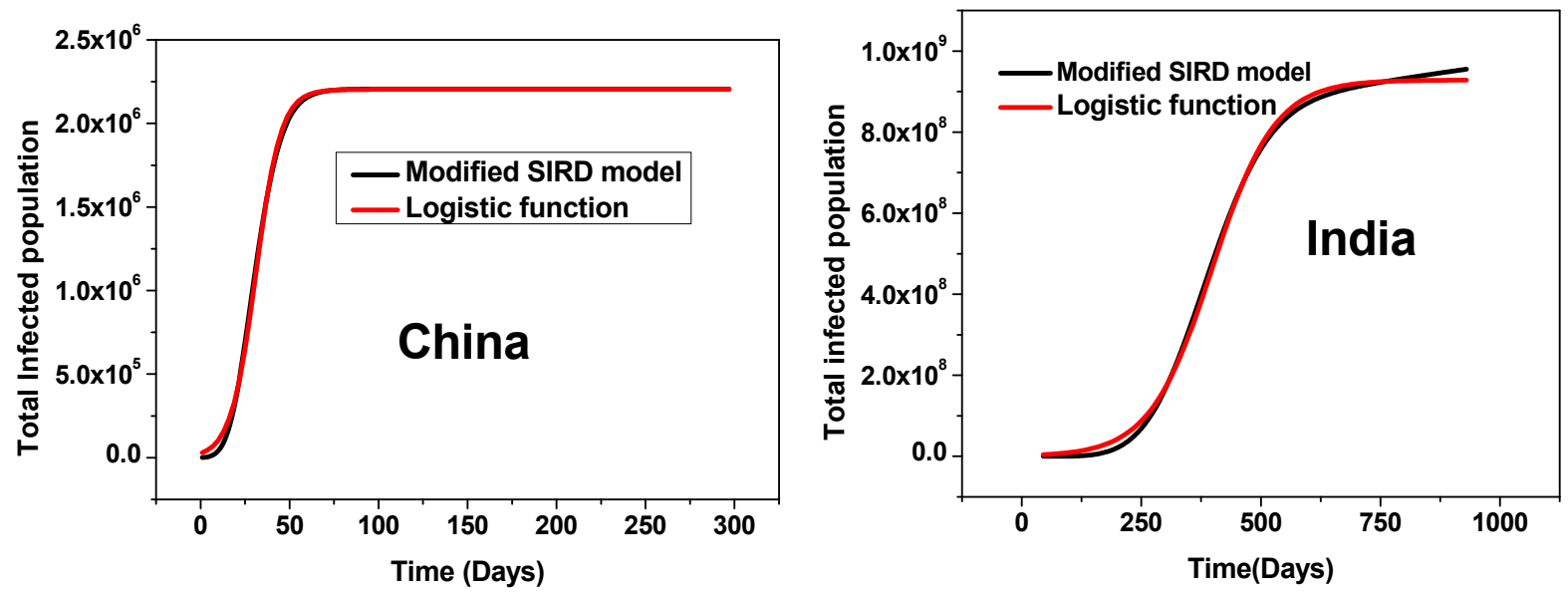

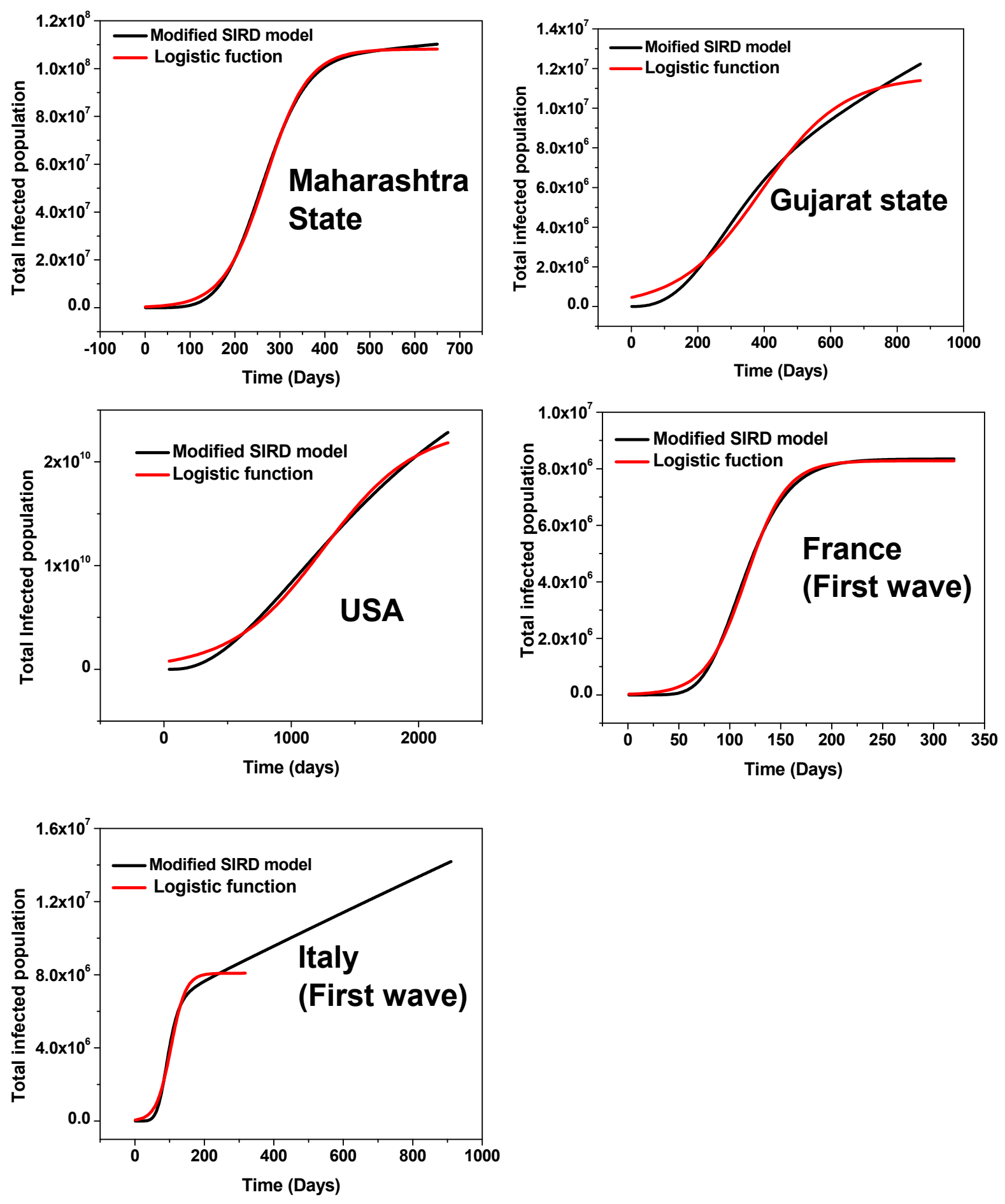

Fig.S4.: Time dependence of total number of infected population for different countries/states is compared with logistic growth function. It is seen that for Italy, USA and Gujarat state of India the variation significantly deviates from logistic growth behavior. 